\section{Qualitätssicherung Akupunktur wird für zwei Jahre ausgesetzt}

_ KBV und Kassen haben die Dokumentationsprüfungen für die Akupunkturbehandlung chronisch schmerzkran-

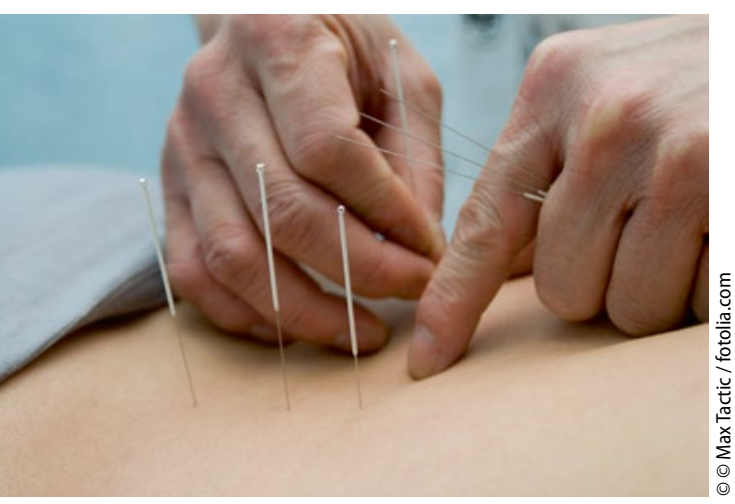

Akupunktur: Bisweilen zahlt die Kasse. ker Patienten bis zum 31. Dezember 2017 ausgesetzt. Die Partner des Bundesmantelvertrags haben sich darauf verständigt, die vorgeschriebenen Dokumentationsprüfungen ab 1. Januar 2016 für zunächst zwei Jahre auszusetzen. Den einzelnen KVen steht es aber frei, die Überprüfung auf freiwilliger Basis weiterhin durchzuführen.

\section{MMW-KOMMENTAR}

Die Qualitätssicherungsvereinbarung zur Akupunktur bei chronisch schmerzkranken Patienten nach § 135 Abs. 2 SGB V, kurz: QSVereinbarung Akupunktur, sieht in $\S 6$ eine jährliche, stichprobenartige Überprüfung der Dokumentationen von Akupunkturbehand-

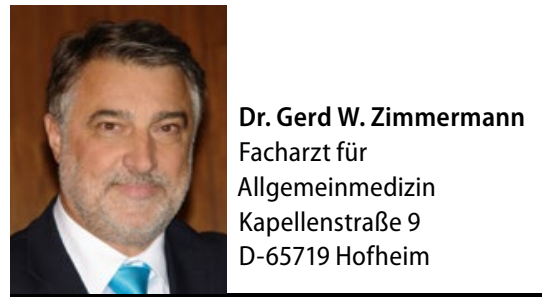

lungen vor. Dabei wurden in den letzten Jahren bundesweit jeweils etwa 10.000 Fälle im Rahmen von Erstprüfungen von den QSKommissionen begutachtet. In einigen KVen gab es auch Wiederholungsprüfungen. Obgleich in etwa 10-20\% der Fälle Beanstandungen ausgesprochen wurden, zeigte sich über die Jahre keine signifikante Veränderung bzw. Verbesserung. Auch setzten die KVen nur in wenigen Einzelfällen weitere Maßnahmen oder Konsequenzen durch, etwa ein Kolloquium oder einen Widerruf der Genehmigung. Das ist der Grund, warum die Prüfungen nun zunächst ausgesetzt wurden. Die Kassen wollen allerdings bis zum 30. Juni 2017 über die Fortführung der Prüfungen oder ggf. weitere Maßnahmen beraten.

\title{
Arthroskopie-Überweisung bei Gonarthrose fällt weg
}

_ Nach einem Beschluss des Gemeinsamen Bundesausschusses (G-BA) wird die Kniegelenksarthroskopie zur Behandlung von Patienten mit Gonarthrose künftig deutlich eingeschränkt. Der Eingriff ist dann nur noch in Ausnahmefällen zulasten der gesetzlichen Krankenversicherung berechnungsfähig. Diese neue Regelung gilt im vertragsärztlichen und im stationären Sektor.

\section{MMW-KOMMENTAR}

Ausgangspunkt dieser Neuregelung war ein Antrag des GKV-Spitzenverbandes an den $G-B A$, diese Leistungen zu überprüfen. Der G-BA beauftragte daraufhin das Institut für Qualität und Wirtschaftlichkeit (IQWiG) mit der systematischen Bewertung der wissen- schaftlichen Unterlagen. Nach deren Erkenntnissen konnte letztlich kein Anhaltspunkt für den patientenrelevanten Nutzen der Arthroskopie bei Gonarthrose gefunden werden. Der G-BA beschloss daraufhin, die Leistungspflicht der Krankenkassen deutlich einzuschränken. Künftig dürfen arthroskopische Eingriffe bei Patienten mit Gonarthrose nur in besonderen Fällen veranlasst bzw. durchgeführt werden, nämlich nach Traumen, bei akuter Gelenksblockade, oder bei einer meniskusbezogenen Indikation, wenn die bestehende Gonarthrose lediglich als Begleiterkrankung anzusehen ist. Die neue Regelung soll am 1. April 2016 in Kraft treten. Spätestens ab diesem Zeitpunkt muss die Diagnose auf der Überweisung eine der genannten Indikationen enthalten.

\section{Letzte Meldung!}

Mit dem Asylverfahrensbeschleunigungsgesetz hat der Gesetzgeber im § 264 SGB V die Ausgabe von elektronischen Gesundheitskarten (eGK) an Empfänger von Gesundheitsleistungen nach den $\S \S 4$ und 6 AsylbLG - also an Anspruchsberechtigte mit weniger als 15 Monaten Aufenthaltsdauer - vorgeschrieben, wenn die Kommunen dies wünschen. Dieser Personenkreis ist auf dem Speicherchip der eGK besonders gekennzeichnet. Ab dem 1. Januar 2016 können nun die Krankenkassen eGKs mit der Kategorie „Besondere Personengruppe 9" an diese Anspruchsberechtigten ausgeben.

Für die niedergelassenen Ärzte ist das natürlich relevant. Die zertifizierten Praxisverwaltungssysteme (PVS) müssen diese „Flüchtlingse-Cards" ab Januar verarbeiten können. Diese neue Funktion ist deshalb Bestandteil der zum Jahreswechsel zur Verfügung gestellten Updates. Im PVS soll dann auch der jeweilige Hinweis auf den eingeschränkten Leistungsanspruch bei dieser „Besonderen Personengruppe" angezeigt werden. 